\title{
Self-administration of intravenous C1 esterase inhibitor in hereditary angioedema
}

\author{
Caroline Rizk MD, Jacob Karsh MDCM, Stephanie Santucci RN, William Yang MD
}

$\mathrm{H}$ ereditary angioedema is a rare autosomal dominant disorder that results in episodes of acute edema in various organs, including the gastrointestinal tract, skin and larynx. It is estimated to affect about 1 in $50000-100000$ people. $^{1}$ The symptoms, including abdominal pain, laryngeal edema and subcutaneous edema, usually begin in childhood and persist throughout life with unpredictable severity. Hereditary angioedema is classified into 3 subtypes: type I, caused by a congenital deficiency of $\mathrm{C} 1$ esterase inhibitor (C1-INH); type II, characterized by dysfunctional $\mathrm{C} 1-\mathrm{INH}$; and type III, an estrogen-dependent form with normal quantitative and functional $\mathrm{C} 1-\mathrm{INH} .{ }^{1} \mathrm{C} 1$ esterase inhibitor plays a role in the complement, fibrinolytic, clotting and kinin pathways. ${ }^{1}$ Without $\mathrm{C} 1-\mathrm{INH}$, activation of these pathways can occur, resulting in bradykinin release and flares of edema.

First-line therapy for acute episodes of type I and II hereditary angioedema is replacing the missing protein with $\mathrm{C} 1$-INH concentrate, which halts the progression of the clinical manifestations within 20 minutes. $^{2} \mathrm{C} 1$ esterase inhibitor is administered intravenously, limiting its use to in hospital, which can delay access to treatment. Self-administration of C1-INH at the first sign of an acute attack is an intuitive intervention to hasten recovery and quickly reduce symptoms, and it has the potential to decrease the number of yearly hospital admissions for affected people. Because of the rarity of hereditary angioedema, emergency department staff may not immediately recognize the symptoms of an acute attack, and patients may not be triaged appropriately. Some hospitals may not stock C1-INH, which may result in treatment delay, the need for higher doses to adequately treat the attack and, as a consequence, higher rates of hospital admissions and severe adverse events, including death. ${ }^{3}$

\section{How does self-administration work?}

Patients keep a supply of C1-INH in their possession (stored at $2^{\circ} \mathrm{C}-25^{\circ} \mathrm{C}$ ). The product consists of a freeze-dried powder that needs to be reconstituted and is stable up to 30 months. Patients should self-administer C1-INH at the earliest sign of an acute attack, which may manifest as abdominal pain, laryngeal edema or subcutaneous edema.

Before beginning home therapy, patients should receive training in sterile technique and the use of equipment for home administration and be given information about the indications for infusion, how to manage emergencies and when to present to an emergency department. This is ideally conducted in a specialized clinic with a multidisciplinary team that includes physicians and nurses; this team facilitates organizing venous access, patient training and regular follow-up. Options for venous access include a peripherally inserted central catheter line, port-acath catheter or self-venipuncture. Patients should still seek medical attention after treatment if they experience a laryngeal attack.

\section{Who may benefit from self-administration?}

Anyone who requires frequent $\mathrm{C} 1$-INH could be eligible, but home treatment would be most

\author{
Competing interests: \\ William Yang has served as \\ a member of national and \\ regional advisory boards for \\ CSL Behring. He has \\ received research grants for \\ clinical trials on acute \\ attacks of hereditary \\ angioedema (not involving \\ self-administered C1-INH) \\ and has received \\ unrestricted educational \\ grants. No competing \\ interests declared by \\ Caroline Rizk, Jacob Karsh \\ and Stephanie Santucci.
}

This article has been peer reviewed.

\section{Correspondence to: Caroline Rizk, carolinerizk@gmail.com}

CMAJ 2013. DOI:10.1503 /cmaj.121364 
beneficial for patients who experience more than 1 attack every 2 months, those with repeated hospital admissions or emergency department visits, or those who need to travel far to reach a hospital. ${ }^{3}$

At our specialized hereditary angioedema clinic, 2 patients were candidates for selfadministration. One presented to the emergency department at every occurrence of symptoms (about 17 visits/yr). Self-administration proved to be especially convenient after she moved to a different region where $\mathrm{C} 1-\mathrm{INH}$ and appropriate care were not readily available. After beginning self-administration 2 years ago, she has had no further visits to the emergency department. Another patient presented to the emergency department 48 times in 2011 alone. Since starting self-administration, she has had a dramatic reduction in hospital use, presenting to the emergency department only 8 times in 2012, either because her symptoms did not resolve immediately or because of a laryngeal attack.

\section{What are the drawbacks?}

Obtaining venous access, especially for patients with angioedema, poses a challenge. However, in a study involving 43 patients, there was less than a $2 \%$ rate of technical failure with venipuncture. ${ }^{4}$ Beyond minor hematomas and skin irritation at the injection site, there were no adverse events that required medical help. ${ }^{4}$ For patients with indwelling intravenous catheters, line infection, sepsis and deep vein thrombosis are ongoing concerns. ${ }^{5}$ One of our patients was admitted to hospital for central line-related bacteremia.

\section{What is the evidence so far?}

Hereditary angioedema attacks disrupt life, affecting work, school and social activities. A review of 6 studies of C1-INH home therapy identified benefits such as a marked reduction in the time to the resolution of an acute attack, reduced hospital admissions, reduced absences from work or school, and improved quality of life. ${ }^{3}$ The study involving 43 patients by Levi and colleagues ${ }^{4}$ found that the time between the onset of an attack and self-administration of C1-INH was 1.4 hours, compared with 3.4 hours for those who relied on medical professionals $(p=0.015){ }^{4}$ The time to complete resolution of symptoms was 5.9 hours after self-administration, a significant improvement from 13.8 hours with conventional treatment $(p=0.035){ }^{4}$

A small study involving 7 patients examined the effect of self-administration on quality of life using the Dermatology Life Quality Index and the 36-Item Short Form Survey. This study found a significant improvement on both physical and psychological levels. ${ }^{6}$ All patients enrolled in the study felt that they had gained control of their disease and were able to resume a normal work and family life, including travelling, without restrictions caused by hereditary angioedema. $^{6}$

Cost-benefit studies of home therapy for hereditary angioedema have not been done, but studies for hemophilia and antibody therapy have found that home therapy is the most costeffective option, reducing costs by up to $20 \%$ compared with in-hospital treatment. ${ }^{7}$ When patients present late during an acute attack of hereditary angioedema, hospital admission is more probable. ${ }^{3,4,7}$ Home therapy decreases the lifetime economic cost of disrupted education and employment in the young population that this condition tends to affect. ${ }^{6}$

\section{What can we expect in the future?}

Subcutaneous administration of C1-INH is still in the early stages of development. Several other subcutaneous drugs for the treatment of hereditary angioedema have been approved for use in the United States and Europe, but they are not licensed in Canada. Ecallantide, a kallikrein receptor antagonist that reduces bradykinin activity, is not approved for selfadministration. Icatibant, a specific bradykinin receptor antagonist given subcutaneously, has also shown promise. ${ }^{1}$

\section{References}

1. Zuraw BL. Hereditary angioedema. N Engl J Med 2008;359: 1027-36.

2. Farkas H, Jakab L, Temesszentandrási G, et al. Hereditary angioedema: a decade of human $\mathrm{C} 1$-inhibitor concentrate therapy. J Allergy Clin Immunol 2007;120:941-7.

3. Longhurst HJ, Carr S, Khair K. C1-inhibitor concentrate home therapy for hereditary angioedema: a viable, effective treatment option. Clin Exp Immunol 2007;147:11-7.

4. Levi M, Choi G, Picavet C, et al. Self-administration of C1 inhibitor concentrate in patients with hereditary or acquired angioedema caused by C1-inhibitor deficiency. J Allergy Clin Immunol 2006;117:904-8.

5. Rooden CJ, Tesselaar MET, Osanto S, et al. Deep vein thrombosis associated with central venous catheters - a review. $J$ Thromb Haemost 2005; 3:2409-19.

6. Bygum A, Andersen KE, Mikkelsen CS. Self-administration of intravenous C1-inhibitor therapy for hereditary angioedema and associated quality of life benefits. Eur J Dermatol 2009;19:147-51.

7. Rodríguez M, Procupet A, Heras J. Cost-effectiveness analysis of home administration versus hospital administration of intravenous immunoglobulin. Med Clin (Barc) 1991;96:47-51.

Affiliations: From the Department of Medicine (Rizk, Karsh, Yang) and the Allergy and Asthma Research Centre (Santucci, Yang), University of Ottawa, Ottawa Ont.

Contributors: All of the authors acquired the data. Caroline Rizk drafted the manuscript, and all of the authors critically reviewed it for important intellectual content. All of the authors approved the final version submitted for publication. 